\title{
A Combined SAXS/WAXS/XAFS Setup Capable of Observing Concurrent Changes Across the Nano-to-Micrometer Size Range in Inorganic Solid Crystallization Processes
}

\author{
Andrew M. Beale, ${ }^{\dagger}$ Ad M. J. van der Eerden, ${ }^{\dagger}$ Simon D. M. Jacques, ${ }^{\ddagger}$ Olivier Leynaud,, \\ Matthew G. O’Brien, ${ }^{\ddagger}$ Florian Meneau, ${ }^{\perp}$ Sergei Nikitenko, ${ }^{\perp}$ Wim Bras, ${ }^{\perp}$ and Bert M. Weckhuysen*, $†$ \\ Inorganic Chemistry and Catalysis, Utrecht University, Sorbonnelaan 16, 3584 CA Utrecht, The Netherlands, \\ Department of Chemistry, University College London, 20 Gordon Street, London WC1H OAJ, United Kingdom, and \\ DUBBLE@ESRF, Grenoble,F-38043, France
}

Received April 27, 2006; E-mail: b.m.weckhuysen@chem.uu.nl

Crystalline inorganic solids show intriguing properties of importance in many technological applications, such as ferromagnetism, semiconductivity, gas sensing, and catalysis. A great deal of attention has been and continues to be focused on understanding why these materials possess these properties. When such phenomena are fundamentally understood, it becomes possible to improve their performance, and this can potentially lead to the development of new "designer" materials to meet the challenges of the future. However, the concept of a designer solid is still some way from being realized since the critical stages of inorganic solid crystallization are still not sufficiently understood.

Time-resolved in situ experimentation represents the most likely means by which the key stages of inorganic solid crystallization can be unraveled. Many studies using a variety of analytical techniques on a range of samples have been reported in the literature with the area of microporous material crystallization receiving a lot of attention. ${ }^{1-3}$ However, such studies often focus on data acquired using a single technique, which rarely provides all of the necessary information from which new insight can be obtained. This is because changes occur over the molecular (local coordination state), nano (primary units), and microscale (crystallite formation) dimension during crystallization, whereas the techniques are often only able to probe limited size regimes. In other words, with separate in situ studies, it is sometimes difficult to obtain consistent and corroborative information. One way to overcome these limitations is to intelligently combine complementary techniques into one experimental setup, and this approach often yields new insight. Some examples include the observation of primary units in zeolite MFI formation and the importance of a coordination state change of $\mathrm{Co}^{2+}$ before the formation of CoAlPO-5 molecular sieves. ${ }^{2,4}$ In addition, a combination of techniques has several advantages. ${ }^{5}$ One being the possibility to evaluate if the probing technique may influence the state of a sample. Furthermore, you circumvent the need to repeat identical experimental conditions for recording separate measurements; although seemingly a trivial point, it is far from easy to achieve experimentally.

In this communication, we present a novel setup, which combines SAXS, WAXS, and XAFS to study the crystallization processes of inorganic solids in depth. This study aims to follow the changes that occur with time at the molecular, nanoscopic, and crystalline level, with a time resolution in the order of a few minutes. The setup enables us to observe the various stages of zinc-substituted microporous aluminophosphate formation in one experiment and

\footnotetext{
$\dagger$ Utrecht University

¥ University College London

$\perp$ DUBBLE@ESRF.
}

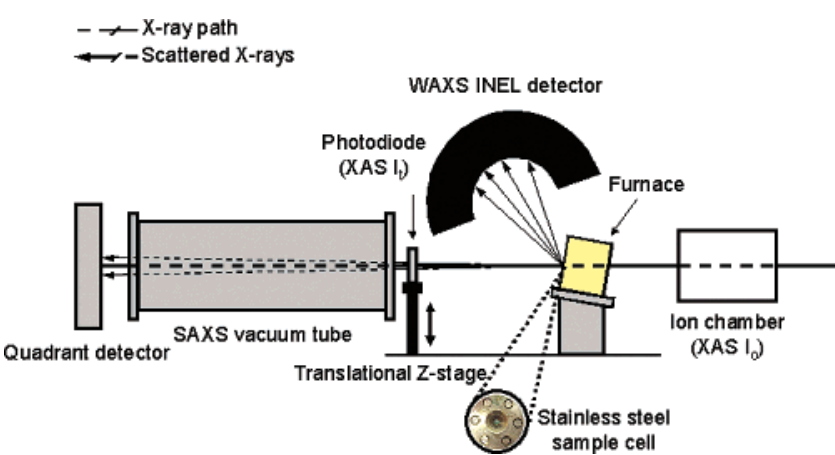

Figure 1. Schematic of the SAXS/WAXS/XAFS multi-technique setup with an enlarged "inside" image of the hydrothermal reactor cell. Data were collected on a bending magnet in transmission mode using a beam with dimensions of $150(\mathrm{H}) \times 3000 \mu \mathrm{m}(\mathrm{V})$ through a sample cell (tilted at ca. $30^{\circ}$ ) containing a gel $\sim 1.5 \mathrm{~mm}$ thick. A wavelength of $1.3098 \AA$ was used for collecting angular dispersive SAXS and WAXS data over a maximum theoretical $k$ range of $0.3<q<2 \mathrm{~nm}^{-1}$ and $2 \theta$ range of $11-45^{\circ}$, respectively. XAFS data were collected from 9.465 to $10.30 \mathrm{keV}$.
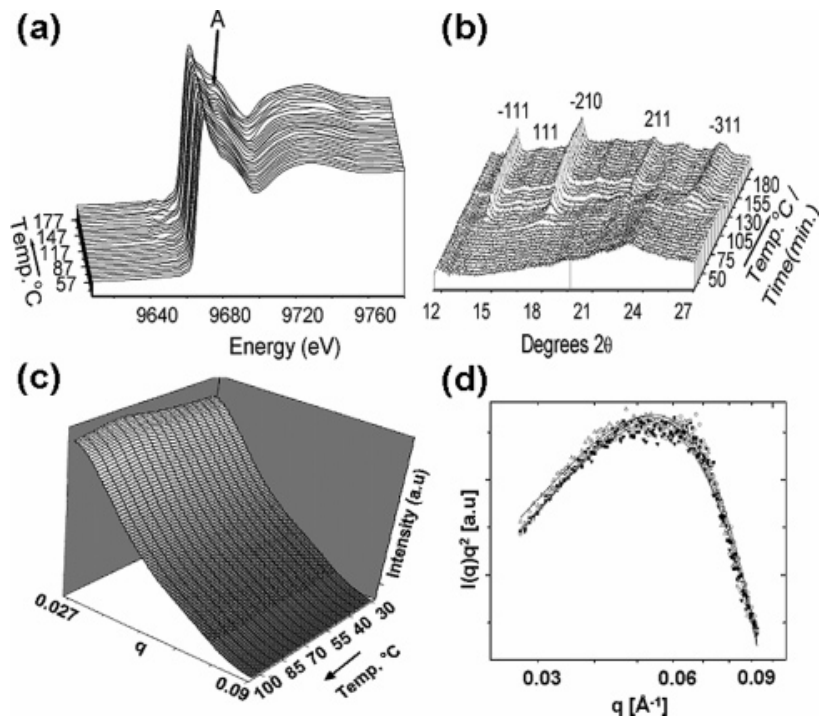

Figure 2. In situ XANES with arrow marking the multiple scattering feature at $\sim 9.685 \mathrm{keV}$ (a), WAXS with reflections indexed to CHA (b), raw SAXS data (c), and $I(q) \times q^{2}-\log (q)$ plots (Kratky type plot) (d) from the SAXS data collected during crystallization.

represents, to the best of our knowledge, the first data of its kind acquired in such a manner.

A scheme of the setup is given in Figure 1. In Figure 2, we show the raw data collected during the heating of the zinc-doped aluminophosphate gel. Data analysis suggested that the initial 

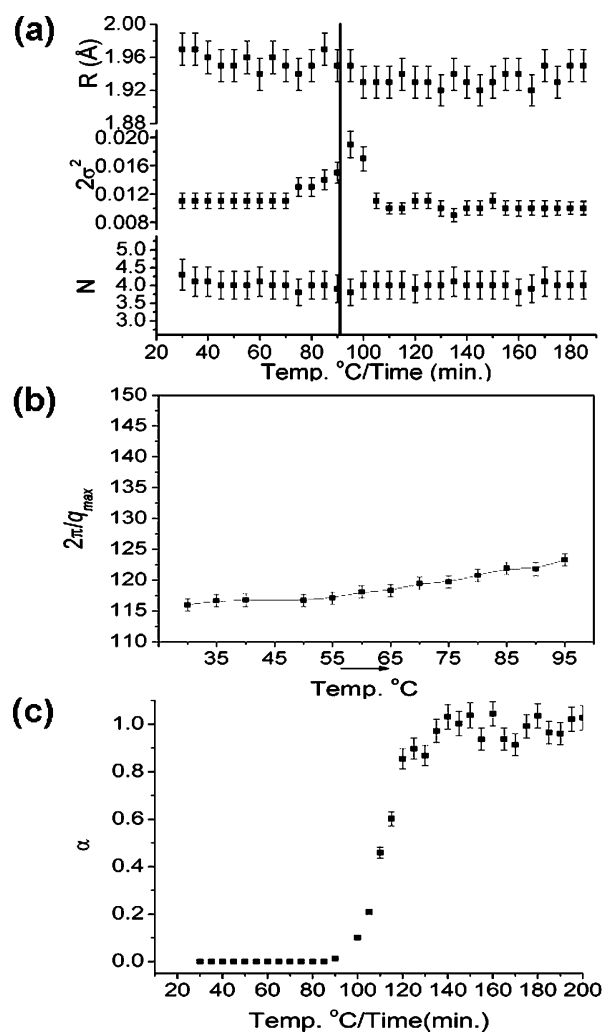

Figure 3. Analysis of the data presented in Figure 2: (a) $\mathrm{Zn}-\mathrm{O}$ EXAFS data, (b) change of $2 \pi / q_{\max }$ position, and (c) extent of crystallization obtained from the reflection intensity change in the WAXS. The increase in the Debye-Waller factor at the onset of crystallization (illustrated by the black line in (a)) is probably artificial and due to fluctuations in sample density as the sample moves in the hydrothermal vessel.

synthesis gel contains predominantly tetrahedral $\mathrm{Zn}^{2+}$ (XAFS edge position $\sim 9.666 \mathrm{keV})^{6}$ and appears diffraction amorphous. The SAXS scattering profile did not appear to contain any marked features, which could be associated with the presence of clear primary units. However, the intensity $I(q)$ closely follows a power law decay $q^{-n}$ (where $n \sim-2$ ), which is slower than the asymptotic behavior of $I(q)$ with $n \sim-4$ predicted by the Porod law for compact particles with sharp interfaces, indicating that this system was more complex. However, by focusing on the $q$ range from 0.025 to $0.1 \mathrm{~nm}^{-1}$ and after removing the leading $q^{-2}$ decay, the $I(q) \times q^{2}$ dependence, the plots exhibit a broad maximum $\left(q_{\max }\right)$ which can be fitted with a Gaussian function and approximated to the inverse of the typical size of possible gel aggregates/precursors leading to AlPO crystallites. The corresponding reciprocal value of $2 \pi / q_{\max }$ approximates to ca. $11.5 \mathrm{~nm}$. When heating to $90{ }^{\circ} \mathrm{C}$, peaks at $13.14,14.65,17.01,21.35$, and $25.28^{\circ} 2 \theta$ started to appear (and eventually grow) in the WAXS pattern, which are indicative for the formation of cubic AlPO-34 (CHA) molecular sieves. ${ }^{7}$ Crystallization of CHA was preceded by small variations in the SAXS profile before a feature in the XANES (a multiple scattering feature) at $9.685 \mathrm{keV}$ was observed to form and grow. Importantly, these changes in the XANES data are consistent with an ordering around the $\mathrm{Zn}^{2+}$ environment and incorporation into the crystalline AlPO-34 structure. Analysis of these data is shown in Figure 3, where we plot the changes in $2 \pi / q_{\max }$ observed in the SAXS data, the intensity of the $\{-111\}$ reflection in the WAXS, and the results from a simple first shell analysis of the EXAFS data during heating. Growth of the final ZnAlPO-34 appeared to be characterized by two stages: a gradual shift to larger $2 \pi / q_{\max }$ values from $\sim 11.5$ to $\sim 12.3 \mathrm{~nm}$ before crystallization of ZnAlPO-34 began at a temper- ature of $\sim 96{ }^{\circ} \mathrm{C}$, and continued crystal growth (the S-type crystallization curve is typical for $\mathrm{AlPO}_{4}$ materials) ${ }^{2}$ occurred until $\sim 160{ }^{\circ} \mathrm{C}$, at which point the crystallite size had grown from $\sim 12$ to $\sim 54 \mathrm{~nm}$ (estimated from a Scherrer analysis). A Sharp-Hancock analysis of the crystallization curve $(0.15<\alpha<0.8)^{2}$ yielded an Avrami exponent of $n \sim 3.30$, which appears consistent with a phase boundary controlled $3 \mathrm{D}$ growth process with a decreasing nucleation rate. However, in contrast to previous work on SAPO-34 materials, no layered 2D-type intermediate phases were observed. ${ }^{8}$ EXAFS analysis revealed that the $\mathrm{Zn}^{2+}$ species remained four-coordinate, hinting of some initial interaction with the amorphous aluminophosphate since $\left[\mathrm{Zn}\left(\mathrm{H}_{2} \mathrm{O}\right)_{6}\right]^{2+}$ was not seen. ${ }^{6}$

In conclusion, the formation of a ZnAlPO-34 material has been studied using a novel combined SAXS/WAXS/XAFS setup. Most importantly, it was observed that growth occurred via a two-step aggregation/crystallization process. The similarity of the average aggregate size measured by SAXS $(\sim 12.3 \mathrm{~nm})$ and WAXS (also $\sim 12 \mathrm{~nm}$ ) suggested that size retention occurs during crystallization, implying that either critical sized aggregates form in the gel structure or else a sort of amorphous-to-crystalline transition leads to $\mathrm{CHA}$ formation. ${ }^{3}$ The growth of this crystalline phase began in preference of AFI despite the synthesis gel and conditions being typical of those used previously for AFI formation. ${ }^{2} \mathrm{Zn}^{2+}$ ions, therefore, appear to both act as nucleating agents and exert some structure directing effect for the formation of the CHA phase. Clearly, this setup is capable of providing a lot of congruous information in one experiment, making it a new and powerful tool for studying crystallization processes of molecular sieves. However, its application need not be restricted to this field alone since it could also provide information on the assembly processes of other crystalline and nanostructured materials. ${ }^{9}$

Acknowledgment. The authors acknowledge NWO/FWO for DUBBLE beamtime. Dr. Tom Blanton is thanked for the silver behenate sample, and Dr. Michel Koch for setting up the quadrant detector. B.M.W. acknowledges NWO-CW for a VICI grant.

Supporting Information Available: Further details on the setup and its operation, data analysis methods, and sample preparation. This material is available free of charge via the Internet at http://pubs.acs.org.

\section{References}

(1) (a) Greaves, G. N.; Bras, W.; Oversluizen, M.; Clark, S. M. Faraday Discuss. 2003, 122, 299. (b) Walton, R. I.; Norquist, A.; Smith, R. I.; O'Hare, D. Faraday Discuss. 2003, 122, 331. (c) Walton, R. I. Chem Soc. Rev. 2002, 31, 230 .

(2) (a) Grandjean, D.; Beale, A. M.; Petukhov, A. V.; Weckhuysen, B. M. J. Am. Chem. Soc. 2005, 127, 14454. (b) Weckhuysen, B. M.; Baetens, D. Schoonheydt, R. A. Angew. Chem., Int. Ed. 2000, 39, 3419.

(3) (a) Cundy, C. S.; Cox, P. A. Microporous Mesoporous Mater. 2005, 82, 1. (b) Rimer, J. D. J.; Fedeyko, M.; Vlachos, D. G.; Lobo, R. F. Chem.Eur. J. 2006, 12, 2926.

(4) (a) de Moor, P. P. E. A.; Beelen, T. P. M.; van Santen, R. A.; Tsuji, K.; Davis, M. E. Chem. Mater. 1999, 11, 36. (b) de Moor, P. P. E. A.; Beelen, T. P. M.; van Santen, R. A.; Beck, L. W.; Davis, M. E. J. Phys. Chem. $B$ 2000, 104, 7600. (c) Sankar, G.; Thomas, J. M.; Rey, F.; Greaves, G. N. J. Chem. Soc., Chem. Commun. 1995, 2549.

(5) (a) Weckhuysen, B. M. Nature 2006, 439, 548. (b) Tinnemans, S. J.; Mesu, J. G.; Kervinen, K.; Visser, T.; Nijhuis, T. A.; Beale, A. M.; Keller, D. E.; van der Eerden, A. M. J.; Weckhuysen, B. M. Catal. Today 2006, 113,3 .

(6) Waychunas, G. A.; Fuller, C. C.; Davis, J. A.; Rehr, J. J. Geochim Cosmochim. Acta 2003, 67, 1031.

(7) Treacy M. M. J.; Higgins J. B. Collection of Simulated XRD Powder Patterns for Zeolites, 4th ed.; Elsevier: Amsterdam, 2001

(8) (a) Vistad, Ø. B.; Akporiaye, D. E.; Lillerud, K. P. J. Phys. Chem. B 2001, 105, 12437. (b) Vistad, Ø. B.; Akporiaye, D. E.; Taulelle, F.; Lillerud, K. P. Chem. Mater. 2003, 15, 1639. (c) Pastore, H. O.; Coluccia, S.; Marchese, L. Annu. Rev. Mater. Res. 2005, 25, 351.

(9) (a) Liu, H. J.; Niu, Y. H.; Han, M.; Liu, Q.; Xu, Z.; Hong, J. N.; Ma, X. Nanotechnology 2005, 16, 2908. (b) Ki, C. D.; Emrick, T.; Chang, J. Y. Adv. Mater. 2005, 17, 230.

JA062580R 